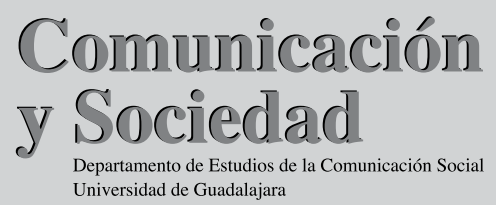

\title{
Enfoques teóricos y estrategias metodológicas en la investigación empírica de audiencias televisivas en América Latina: 1992-2007
}

\author{
JOSÉ CARLOS LOZANO Y LORENA FRANKENBERG ${ }^{1}$
}

El artículo reporta avances de una revisión de estudios empíricos de audiencias televisivas en América Latina, publicados entre 1992 y 2007 en las principales revistas científicas del campo. En 51 trabajos, se analiza la claridad conceptual, los marcos teóricos, las estrategias metodológicas y se identifican las tendencias y omisiones en la investigación sobre recepción televisiva. En los hallazgos destacan la supremacía de los estudios culturales como enfoque teórico, la vida cotidiana y las mediaciones familiares como objetos de estudio y la falta de solidez en la metodología. Se presenta, además, un panorama sobre las fortalezas y debilidades de estas investigaciones, se subraya la necesidad de cuidar el rigor metodológico y se sugiere enfatizar el análisis de lecturas ideológicas.

PALABRAS CLAVE: audiencias televisivas, estudios culturales, recepción televisiva, investigación empírica de audiencias
This paper reports the findings of a review of 51 Latin American empirical studies on television audiences published between 1992 and 2007 in the most important journals in the region. The analysis compares the studies according to their theoretical approach, research techniques and type of television content. Findings show that cultural studies is the most popular theoretical approach, that television and daily life, and the mediations are the topics covered the most, and that many studies still lack a solid methodological base. The paper presents a diagnosis of the strengths and weaknesses of current Latin American empirical research on television audiences and points out the need for more methodological rigor and emphasis on ideological readings.

KEY WORDS: television audiences, cultural studies, television reception, empirical audience research.

1 Tecnológico de Monterrey, Campus Monterrey.

Correos electrónicos: iclozano@itesm.mx / lfrankwnberg@intercable.net. 
En 1994, McAnany y La Pastina dieron a conocer los resultados de una revisión y crítica metodológica de diversos estudios de audiencias de telenovela en América Latina. Los hallazgos se basaban en 26 trabajos empíricos, y aunque reconocían el crecimiento de los estudios de este tipo, advertían sobre sus debilidades, en especial en cuanto a su rigor metodológico. Los autores destacaban la importancia de la telenovela en América Latina como género dominante en las preferencias de las audiencias televisivas y admitían su preocupación sobre las consecuencias sociales y culturales de dicho fenómeno.

Los investigadores latinoamericanos, según los autores, estaban conscientes de la importancia que tenía la televisión por su impacto en la sociedad. Sin embargo, los estudios empíricos de audiencias habían sido relativamente escasos y la literatura, hasta 1994, remitía, en la mayoría de los casos, solamente a las investigaciones de Katz, Liebes y Morley.

McAnany y La Pastina concluyeron que gran parte de los trabajos analizados presentaban deficiencias metodológicas debido a la poca experiencia de los investigadores, quienes en su mayoría eran alumnos realizando sus tesis de maestría o sus disertaciones doctorales. Además, los autores contrastaron la escasa publicación de estudios de esta índole en América Latina con la investigación más sistemática sobre telenovelas existente en Estados Unidos. En resumen, la revisión concluyó con un llamado para aumentar la investigación empírica de audiencias y la necesidad de mejorar la calidad y metodología de los trabajos.

En este contexto, la presente revisión tiene como objetivo plantear el panorama en los años posteriores al trabajo de McAnany y La Pastina. El propósito principal de este artículo radica en explorar y evaluar la investigación empírica de audiencias televisivas realizada en América Latina durante los últimos quince años. Nuestro estudio, sin embargo, no pretende ser una réplica al trabajo de estos investigadores y se ha extendido a todos los géneros televisivos abordados en los estudios disponibles. Esto con el fin de ampliar la base de conocimiento que permita a los investigadores de la televisión en general y sus audiencias analizar la situación actual, plantear propuestas y generar sugerencias hacia el futuro. Se han examinado para este propósito los métodos empleados en las investigaciones, sus marcos teóricos, la procedencia de los trabajos, los géneros estudiados y el conocimiento derivado de ellos. 


\section{LOS ESTUDIOS DE RECEPCIÓN EN AMÉRICA LATINA}

Anterior a la década de 1990 los estudios sobre comunicación, en América Latina, se enfocaban principalmente en el análisis crítico de los medios como instituciones, otorgándole muy poca atención a las audiencias. Los investigadores, apoyados en la economía política o en el análisis de contenido, tendían a considerar al receptor como un ente pasivo ante el dominio y la hegemonía de las industrias culturales. Estos planteamientos ignoraban casi por completo el estudio de los procesos de recepción y consumo en las audiencias latinoamericanas. En sociedades dominadas por el neoliberalismo, los teóricos de la dependencia asumían que al comprobarse la existencia de la transnacionalización en el proceso de producción y distribución, se podía colegir la transnacionalización de los procesos de recepción (Lozano, 1990/1991).

Frente a este panorama empezó a surgir, a partir de la segunda mitad de los años ochenta, una nueva perspectiva crítica interesada en los procesos de recepción y consumo de los productos culturales. Los trabajos de García-Canclini (1988), Martín Barbero (1987) y González (1987) apuntaron hacía nuevas consideraciones sobre el aspecto cultural y las mediaciones que utilizan los receptores para interpretar los significados comunicacionales.

A partir de la década de 1990, los trabajos inscritos en esta nueva perspectiva reflejaron la concepción de las audiencias como activas, lo que ya era común en enfoques críticos de otras partes del mundo desde fines de los setenta, pero que por el auge de los paradigmas de la dependencia y del imperialismo cultural no se había extendido en la región. Académicos de los diferentes países latinoamericanos empezaron a generar conocimiento en esta línea siguiendo el liderazgo teórico de Martín Barbero, García Canclini, Orozco y González.

En Argentina, al institucionalizarse los estudios de recepción, se insertó en ellos un abanico de enfoques epistemológicos y teóricos procedentes de las ciencias sociales que entraron al campo como nuevas referencias (Saintout y Ferrante, 2006). En sus trabajos, estos autores exploraron las formas en que los grupos subordinados interactúan y negocian la cultura de masas, puntualizando el desplazamiento del poder en su concepción como imposición hacia la noción de hegemonía y 
resistencia. Sin embargo, en los últimos años, la comunidad académica argentina se ha inclinado más hacia el análisis y ensayo teórico que hacia la investigación empírica. De acuerdo a Saintout y Ferrante, los pocos estudios empíricos de audiencias abordan principalmente la relación entre comunicación y educación, inspirados por los trabajos de Orozco, en México, y el consumo cultural de bienes y su vinculación con la identidad, derivados de los planteamientos de García Canclini.

En Brasil, la producción científica y académica en el área de comunicación apareció en la década de 1970 al implementarse los primeros cursos de posgrado. Las investigaciones pioneras revelaban la influencia de la teoría crítica, la semiología y la teoría de los efectos. Sin embargo, es a partir de los ochenta cuando se acelera la producción de estudios de audiencia que generarían una nueva tendencia en los estudios de comunicación. Este sesgo, de acuerdo a Jacks y Escosteguy (2006), se expresó principalmente en la articulación de los sujetos-receptores con el ámbito más amplio de la cultura. La investigación empírica de audiencias se institucionalizó en el campo académico de ese país en la década de 1990; sin embargo, no ha generado un amplio debate en el campo de la comunicación brasileña comparado con la trayectoria en el medio anglosajón.

En una revisión sobre el estado del arte en Colombia, Martín Barbero y Téllez (2006:58), encontraron una situación similar al resto de América Latina. Los estudios de recepción en ese país surgen en los años ochenta en una concepción ligada a los efectos con base en dos situaciones clave para la sociedad: "la fragilidad de la democracia y el espesor de la violencia". Sin embargo, en los noventa se presenta el desplazamiento de la recepción al consumo, y al finalizar el milenio, según los autores, los niños aparecen como ejes centrales de la investigación. Este nuevo enfoque destaca la importancia de romper con la percepción de la fragilidad y pasividad de las audiencias, en este caso los jóvenes y niños, considerados como los mayores afectados por los efectos "nocivos" de la televisión. Propone, en su lugar, entender sus competencias comunicativas y cambiar la visión de la televisión como nociva por una posición más abierta y flexible, en la que se tome en cuenta ámbitos como la escuela y la familia como principales espacios de socialización y mediación de los contenidos televisivos. 
En el caso de Chile, Fuenzalida (2006:47) ha advertido la importancia de reconceptualizar, desde la mirada de la recepción, los temas clásicos abordados por la comunicación televisiva. Estos temas se ordenan desde el microacontecer comunicacional en el seno del hogar, hasta problemas sociales y políticos. De acuerdo con el reconocido investigador, debe considerarse que en Chile, por la dictadura, los estudios de recepción estuvieron excluidos de la universidad hasta 1990. Fue hasta la creación de programas de posgrado que estas investigaciones comenzaron a desarrollarse. Los estudios iniciales, según Fuenzalida, abordaron los temas espacio temporales de la vida cotidiana con observación etnográfica, obligando la reconsideración teórica de temas como el de la entretención.

Desde la perspectiva de la recepción televisiva, la entretención aparece como un sentimiento actitudinal complejo que se opone al aburrimiento, al desinterés, la desatención, el no-involucramiento; el sentimiento de entretención no es contradictorio, entonces, con información o educación; esto es, no es un sentimiento que anule la cognición, el cual ha sido uno de los grandes temores de occidente (ibidem: 51).

En este sentido, para Fuenzalida la telenovela destaca como género que produce una importante "cultura televisiva", esto es "la cultura transtextual de la audiencia participa en la construcción del sentido textual" (ibidem:52). Se confirma, además, como el género que más suscita la convivencia familiar y social. Asimismo, esta reorientación etnográfica en los estudios de audiencias ha permitido una reconceptualización entre la recepción televisiva en el hogar y la educación y la política. La evaluación de los receptores se orienta a la contribución de estos aspectos en tanto mejoran la calidad de vida experimentada desde el hogar.

En México, la investigación de la recepción televisiva se ha desarrollado a partir de diferentes vetas. Como lo señalan Orozco y Padilla (2005), durante la década de 1980 un estudio realizado en la Universidad Autónoma Metropolitana de Xochimilco contribuyó significativamente a desmitificar el impacto y los efectos de los medios masivos de comunicación permitiendo conceptualizar al sujeto como activo. Los trabajos del Programa Cultura, desarrollados en la Universidad de Colima y liderados por Jorge González y Jesús Galindo, fueron medulares 
para valorar la telenovela como importante producto de cultura popular con fuerte intervención en la conformación de identidad y cultura nacional. Con el concepto "frentes culturales", describieron la existencia de una disputa simbólica mediante la cual cada grupo trataba de imponer o de apegarse a su propia identidad cultural (González, 1987).

Los primeros estudios de recepción en México se inclinaron hacia el área de la educación. A partir de la década de 1980, la investigación exploró la relación de la televisión con las instituciones socializantes de la escuela y la familia. Los análisis reconocían la tendencia hacia una recepción crítica e inteligente por parte de los sujetos. Otra de las temáticas de gran interés en México en cuanto a estudios de recepción televisiva, según Orozco y Padilla, fue la relativa al papel de los noticieros en la comprensión del entorno.

Sus objetivos pretenden comprender cómo la ubicación geopolítica, el sentido de pertenencia nacional, el nivel socioeconómico y el género se ubican como mediaciones en los procesos de recepción de noticias" (2005: 151).

Sin embargo, una perspectiva diferente para estudiar la recepción televisiva se desarrolló a partir de mediados de los noventa en Monterrey, México. Lozano y Martínez, entre otros académicos, han estudiado la recepción de contenidos televisivos extranjeros entre los habitantes del noreste. Entre los hallazgos más consistentes de estas investigaciones se encuentra la clara preferencia de las audiencias por los contenidos televisivos nacionales sobre los contenidos estadounidenses a pesar de la proximidad geográfica de ciudades fronterizas como Nuevo Laredo (Lozano, 1996) y la ciudad más importante de la región, Monterrey (Lozano, 2003; Martínez, 1994) con el estado de Texas. Un grupo de investigadores ha seguido esta misma línea de estudio, explorando los patrones de consumo de televisión extranjera en diversas ciudades, encontrando la misma preferencia por los contenidos locales sobre las producciones de importación en ciudades como Saltillo y Torreón, Reynosa, Tamaulipas y Monterrey, (Chong y Ornelas, 2006; García Álvarez, 2007; Hinojosa et al., 2005; Lozano, en prensa).

La excepción a estas preferencias se presenta en el cine. Los hallazgos de las encuestas aplicadas en las ciudades del noreste de México revelan 
que la audiencia prefiere y consume un gran número de filmes norteamericanos durante su exposición televisiva. Se confirma así la hipótesis de la "proximidad cultural", que afirma que los miembros de la audiencia prefieren contenidos locales sobre los extranjeros excepto en los géneros no producidos en su propia localidad (Straubhaar, 1991). Otra línea de investigación explorada por Lozano (2003) y Gutiérrez (2006) es la decodificación y distanciamiento crítico de las audiencias sobre programas televisivos en particular, tema escasamente explorado por investigadores en el país. Estos académicos, que trabajan en el Centro de Investigación en Comunicación e Información (CINCO) del Tecnológico de Monterrey, han encontrado resultados mixtos sobre la capacidad de las audiencias para distanciarse críticamente de los contenidos televisivos de su preferencia. Los trabajos de Lozano (2001, 2003), por ejemplo, revelan que las audiencias de todas las edades y niveles socioeconómicos pueden cuestionar y negociar los noticieros televisivos, pero parecen incapaces de verbalizar críticas contra sus programas favoritos de ficción.

La familia, como una mediación importante en la recepción televisiva, es otro de los campos más estudiados en México (Orozco, 1992). La vida cotidiana, la configuración de identidades en familia, los estudios de género, la comunicación familiar y la influencia de la madre en los procesos de recepción televisiva en el hogar han sido las principales líneas estudiadas empíricamente.

La investigación de audiencias en los diferentes países latinoamericanos, como se observa en la discusión anterior, tiene una historia muy corta en la mayoría de los casos y se ha centrado en el papel de la televisión en la vida de los televidentes. Diversos académicos, usando distintos enfoques metodológicos y enfocados en sujetos de variadas características, han descrito estas relaciones. La complejidad del objeto de estudio ha llevado a que en México, así como en otros países, se rebase el concepto de recepción. Orozco y Padilla (2005:155), por ejemplo, han propuesto hablar mejor de "televidencia" entendida como

la multidimensionalidad de las interacciones que movilizan las diversas particularidades y temporalidades desde que el sujeto individual y colectivo procesa los discursos y las narrativas, las mitologías y los imaginarios de la televidencia. 
Expuesto así el panorama general sobre la investigación de audiencias televisivas en América Latina, se procede a mostrar el resultado del análisis de los 51 trabajos encontrados en este contexto en las revistas académicas más importantes de la región a partir de 1992. Las preguntas que guiaron esta investigación fueron las siguientes:

1. ¿Cuál es la importancia y presencia de los estudios empíricos de recepción televisiva en América Latina en comparación con otro tipo de trabajos publicados en las revistas seleccionadas?

2. ¿Siguen siendo las telenovelas el principal objeto de estudio de la investigación empírica de audiencias en la región, o esta ha incorporado otros géneros televisivos?

3. ¿Qué tipo de audiencias y temas son los más estudiados en estos trabajos?

4. ¿Qué enfoques teóricos y analíticos predominan al interpretar los hallazgos de estas investigaciones?

5. ¿Quiénes son los académicos más citados en estos trabajos? ¿Han realizado estos investigadores estudios empíricos de recepción televisiva en los últimos 15 años? ¿Siguen trabajando estas mismas líneas de investigación?

6. ¿Cuáles son las técnicas cualitativas o cuantitativas más utilizadas y con qué tamaño de muestras? ¿Indican los tamaños de las muestras posibles fallas en la generalización y confiabilidad de los hallazgos?

7. ¿Qué tan detallada y rigurosa es la metodología empleada en estos estudios?

\section{MÉTODO}

El análisis comprende 51 trabajos publicados desde 1992 en revistas latinoamericanas de comunicación y ciencias afines. La revisión se realizó buscando los estudios inscritos en esta temática, que estuvieran disponibles en las revistas especializadas más importantes de América Latina en su versión electrónica, y algunas en papel propiedad del CINCO.

La base de datos utilizada consta de 17 revistas que sumaron un total de 374 números revisados. Se buscaron los textos completos de los artícu- 
los en las bases de datos en línea Redalyc (Red de Revistas Científicas de América Latina, el Caribe, España y Portugal), Revcom (Revistas Eletrônicas de Ciências da Comunicação, de Brasil), la base de datos del Centro de Documentación del CONEICC en el ITESO, el portal INFOAMERICA y los sitios originales de las revistas Diálogos de la Comunicación, Razón y Palabra, Global Media Journal en Español, ZER y Palabra-Clave. En papel se consultaron todos los ejemplares de 1992 a la fecha de Comunicación y Sociedad, de Guadalajara y el Anuario de Investigación de la Comunicación CONEICC. De varias revistas, como las brasileñas, sólo se revisaron los números que estaban disponibles en el sitio Revcom, los cuales, en la mayoría de los casos eran muy pocos. Los hallazgos sobre este país, así, no pueden tomarse como una muestra representativa debido al significativo número de ediciones pendientes de analizar.

La limitación de esta muestra radica en la falta de acceso a algunas publicaciones académicas de los diversos países, así como la carencia del texto completo disponible en red. Debe considerarse que este trabajo está en proceso, ya que la búsqueda de las ediciones faltantes de cada revista seleccionada se sigue realizando. Asimismo, falta incluir otras revistas, sobre todo de países como Venezuela, Colombia, Perú, Chile, Argentina, Uruguay, etc., que no aparecían en ninguna de las bases de datos consultadas. Para ello, actualmente se está trabajando con un grupo de colegas en esos países para la segunda fase de esta investigación, la cual incluirá dichas publicaciones. De cualquier forma consideramos que las principales revistas han sido incluidas y que el número de estudios revisados alcanza a reflejar las principales tendencias en la investigación latinoamericana de audiencias televisivas publicadas en las revistas académicas de la región. Asimismo, cabe hacer notar que la ausencia de investigaciones empíricas de audiencias en las revistas y bases electrónicas consultadas hacen que su impacto en el campo de investigación latinoamericano sea muy limitado.

Es importante señalar que parte de la investigación empírica de audiencias televisivas se publica en libros, capítulos de libros y cuadernos de investigación que no se tomaron en cuenta en este estudio debido a la dificultad en identificarlos y consultarlos.

Cada uno de los 51 trabajos incluidos fue analizado detalladamente para alimentar una base de datos compuesta por campos donde se 
especificaba el nombre de la investigación, el autor, la publicación, el país de procedencia, el año, la técnica utilizada, el enfoque teórico, así como los autores citados en la obra, la muestra, la claridad de las preguntas de investigación y la metodología expuesta. A partir de esa base de datos, se procedió a analizar cuantitativa y cualitativamente la información recabada.

\section{RESULTADOS}

¿Cuál es la importancia y presencia de los estudios empíricos de recepción televisiva en comparación con otro tipo de trabajos publicados en las revistas seleccionadas? La tabla 1 muestra que sólo 14\% de todos los artículos publicados en 374 números de las 17 revistas seleccionadas eran investigaciones empíricas de recepción televisiva. Si otros tipos de estudios de campo son incluso menos populares, como parece ser el caso, este hallazgo podría indicar un patrón en las revistas latinoamericanas de favorecer la publicación de ensayos teóricos sobre los trabajos empíricos. O quizás sólo refleje la situación actual de la academia latinoamericana de comunicación, más propensa al trabajo teórico que al empírico por la falta de fondos y la escasa preparación de los investigadores para el trabajo de campo (Lozano, 2007:87-96).

\section{De la telenovela a otros géneros televisivos}

McAnany y La Pastina (1994:828-849) encontraron, entre 1970 y 1993, 26 trabajos en revistas académicas, libros y tesis de posgrado que analizaban la recepción de telenovela. En la presente revisión, sólo $12(23 \%$ de los 51 trabajos revisados, se concentraron específicamente en la revisión de este género televisivo (tabla 2). Más de la mitad de los artículos revisaban el consumo televisivo en general, algunos incluso estudiando varios géneros al mismo tiempo. Esto podría constituir una tendencia de la investigación más reciente por tomar en cuenta los distintos tipos de contenidos que consumen diferentes tipos de audiencias, algunos estudios enfocándose en el origen geográfico de los programas preferidos (De la Garza, 1997; Huerta, 2005; Huerta y Cerda, 2002; Lozano, 1994; Lozano, 2003); otros revisando la diversidad de contenidos preferidos 


\section{TABLA 1}

NÚMERO DE ESTUDIOS EMPÍRICOS DE AUDIENCIAS TELEVISIVAS PUBLICADOS EN REVISTAS ACADÉMICAS DE AMÉRICA LATINA ENTRE 1992-2007 POR TÍTULO

\begin{tabular}{|c|c|c|c|c|c|}
\hline$\stackrel{\circ}{\stackrel{B}{\Xi}}$ & $\stackrel{n}{\pi}$ & 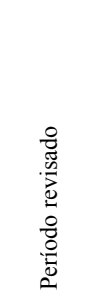 & 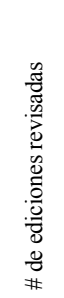 & 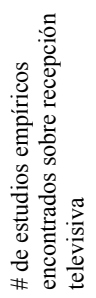 & 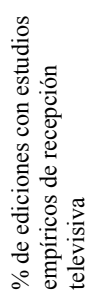 \\
\hline Comunicación y Sociedad & México & $92-07$ & 34 & 14 & 41 \\
\hline Anuario de Inv. CONEICC & México & $94-06$ & 13 & 10 & 77 \\
\hline \multicolumn{6}{|l|}{ Diálogos de la } \\
\hline Comunicación & Perú & $92-07$ & 42 & 5 & 12 \\
\hline Culturas Contemporáneas & México & $92-06$ & 27 & 4 & 15 \\
\hline Zer & España & $96-07$ & 21 & 4 & 19 \\
\hline Palabra-Clave & Colombia & $96-01$ & 16 & 3 & 19 \\
\hline Intercom & Brasil & 00-07 & 14 & 2 & 14 \\
\hline Contemporánea & Brasil & 03-06 & 5 & 2 & 40 \\
\hline Nombre Falso & Argentina & $01-07$ & 9 & 2 & 22 \\
\hline Razón y Palabra & México & $96-07$ & 58 & 1 & 2 \\
\hline FAMECOS & Brasil & 01-07 & 20 & 1 & 5 \\
\hline Fronteiras & Brasil & 04-07 & 9 & 1 & 11 \\
\hline Global Media Journal & México & 04-07 & 7 & 1 & 14 \\
\hline Comunicação \& Sociedade & Brasil & 02-04 & 5 & 0 & 0 \\
\hline Contracampo & Brasil & $02-03$ & 2 & 0 & 0 \\
\hline Comunicación & C. Rica & $00-06$ & 11 & 0 & 0 \\
\hline \multicolumn{6}{|l|}{ Rev Mexicana de Ciencias } \\
\hline Políticas y Sociales & México & $92-06$ & 45 & 1 & 2 \\
\hline Total & & & 374 & 51 & $14 \%$ \\
\hline
\end{tabular}

Nota: El número total de ediciones 2002 y 2007 de algunas revistas no fueron revisadas porque no se encontraban disponibles en las bases de datos y bibliotecas utilizadas en este estudio. La revista española ZER fue incluida por los 4 estudios latinoamericanos publicados en ella. 


\section{TABLA 2}

PORCENTAJE DE ESTUDIOS POR GÉNERO TELEVISIVO

\begin{tabular}{|c|c|c|}
\hline Género & $\mathrm{f}$ & $\%$ \\
\hline Varios & 27 & 52.9 \\
\hline Telenovelas & 12 & 23.5 \\
\hline Noticias & 8 & 15.7 \\
\hline Entretenimiento & 1 & 2.0 \\
\hline Series & 1 & 2.0 \\
\hline Ninguna & 1 & 2.0 \\
\hline Reality shows & 1 & 2.0 \\
\hline Total & 51 & $100 \%$ \\
\hline
\end{tabular}

por segmentos específicos de la audiencia como los jóvenes (Chavero y García Muñoz, 2005; González Hernández, 2006; López, 2003, 2004; Martínez, 1994; Orozco y Viveros, 1996; Renero, 1996;) e incluso otros más interesados en las mediaciones familiares en el proceso de ver televisión (Cornejo, 1992; Guadarrama, 1999; Renero, 1992, 1995 y 1997; Vega, 2005).

La recepción de noticias es un área de creciente interés, particularmente en una región en la cual la investigación sobre periodismo se ha centrado en los contenidos informativos o en los propios periodistas. El hecho de que $16 \%$ de los estudios revisados dedique su análisis a la recepción de noticieros, revela la atención que este género está recibiendo por algunos académicos latinoamericanos. En contraste, géneros como las series televisivas y los reality shows, pese a la importante cantidad de producciones e importaciones, han sido casi ignorados por los estudios dedicados a analizar el consumo.

\section{Jóvenes y adolescentes}

Los trabajos empíricos sugieren que los investigadores latinoamericanos están centrando sus esfuerzos en analizar, en su mayoría, la recepción televisiva entre jóvenes y adolescentes (tabla 3). Los adultos también representan un segmento considerable en los trabajos de investigación, mientras que los niños y las mujeres no han atraído suficiente atención de los pocos académicos haciendo trabajo de 


\section{TABLA 3}

PORCENTAJE DE ESTUDIOS DE ACUERDO AL TIPO DE AUDIENCIA

\begin{tabular}{lcc}
\multicolumn{1}{r}{ Audiencia } & f & $\mathbf{\%}$ \\
\hline Jóvenes & 11 & 21.6 \\
Adultos & 8 & 15.7 \\
Indefinido & 7 & 13.7 \\
Familia & 7 & 13.7 \\
Niños & 5 & 9.8 \\
Varios & 4 & 7.8 \\
Adolescentes & 4 & 7.8 \\
Mujeres & 4 & 7.8 \\
Hombres & 1 & 2.0 \\
Total & $\mathbf{5 1}$ & $\mathbf{1 0 0 \%}$ \\
\hline
\end{tabular}

campo. La selección de adultos y de jóvenes, sin embargo (Bonilla, 1998; Cerda, 2001; Chavero y García Muñoz, 2005; Dalmonte, 2006; De Jesús y Patriota, 2007; De la Garza, 1997; Diez Martínez, Miramontes y Sánchez, 2000; González, 2006; Marques y Rocha, 2006; López, 2003/2004; Lozano, 1994; Martínez, 1994; Orozco y Viveros, 1996; Renero, 1996) podría ser resultado de procedimientos de conveniencia y no necesariamente de preocupaciones sociales o teóricas. Los investigadores encuentran más fácil el acceso a este tipo de audiencias en las escuelas o en sus propias universidades. Otro factor influyente es el hecho de que los académicos no cuentan con fondos para realizar estas investigaciones y trabajan con fuertes sobrecargas de materias, lo cual los obliga a trabajar con muestras accesibles en sus propias instituciones o ciudades. De cualquier forma, los estudios proveen evidencia importante sobre las complejas interacciones entre algunos de los grupos sociales más sensibles con respecto a la televisión.

\section{La supremacía de los estudios culturales}

Estudios culturales es, por mucho, la perspectiva teórica que inspira la mayoría de los trabajos empíricos sobre recepción televisiva en América Latina. La tabla 4 muestra que más de la mitad de las investi- 
gaciones revisadas se basa en esta aproximación y la tabla 5 confirma esta conclusión al identificar a Morley, Orozco, Martín Barbero, García Canclini y James Lull como los autores más citados. Este hallazgo no es raro en una región como América Latina, donde los estudios críticos como la teoría de la dependencia y el imperialismo cultural primero, y los estudios culturales después, se han consolidado en las últimas décadas.

\section{TABLA 4}

PORCENTAJE DE ESTUDIOS POR ENFOQUE TEÓRICO

\begin{tabular}{lcc}
\multicolumn{1}{c}{ Enfoque } & $\mathrm{f}$ & $\%$ \\
\hline Estudios culturales & 30 & 58.8 \\
No identificado & 9 & 17.6 \\
Análisis de recepción & 3 & 5.9 \\
Otro & 3 & 5.9 \\
Usos y gratificaciones & 2 & 3.9 \\
Ecléctico & 2 & 3.9 \\
Educación para los medios & 1 & 2.0 \\
Varios & 1 & 2.0 \\
Total & $\mathbf{5 1}$ & $\mathbf{1 0 0 \%}$ \\
\hline
\end{tabular}

Las valiosas aportaciones de autores como Martín Barbero, García Canclini, Orozco, González y Fuenzalida, entre otros, en la década de 1980 en el campo genéricamente identificado como estudios culturales ${ }^{2}$, siguen siendo el fundamento teórico para la mayoría de estos trabajos

2 La ubicación de autores como Orozco y Fuenzalida dentro del enfoque de los estudios culturales debe ser considerada con cuidado, ya que no comparten todas las bases conceptuales y metodológicas que caracterizan a los "cultural studies" anglosajones. Quizás, ambos podrían ubicarse mejor bajo el término de "reception analysis" (ver Jensen y Rosengren, 1990) o el de "estudios de medios" o "media studies" (Orozco y Padilla, 2005: 155). Los planteamientos de Martín Barbero y de García Canclini, igualmente, podrían tener diferencias importantes con algunos de los presupuestos de la corriente europa de los estudios culturales. 


\section{TABLA 5}

NÚMERO DE ARTÍCULOS QUE CITAN A UN AUTOR MÁS DE DOS VECES DENTRO DEL TEXTO

\begin{tabular}{lc}
\multicolumn{1}{c}{ Autor } & $\mathrm{f}$ \\
\hline David Morley & 15 \\
Guillermo Orozco & 15 \\
Jesús Martín Barbero & 12 \\
Néstor García Canclini & 8 \\
James Lull & 8 \\
Klaus B. Jensen & 5 \\
Jorge González & 4 \\
Ien Ang & 3 \\
Gilberto Giménez & 3 \\
Valerio Fuenzalida & 2 \\
Stuart Hall & 2 \\
Juan José Igartua y Carlos Muñiz & 2 \\
Michael Morgan y James Shanahan & 2 \\
Roger Silverstone & 2 \\
Otros autores con dos o más menciones en & 53 \\
un solo artículo & \\
Total & 136 \\
\hline
\end{tabular}

en la región. Sin embargo, resulta interesante observar que el trabajo más reciente sobre audiencias no ha incorporado aún las contribuciones de los nuevos investigadores empíricos contemporáneos. Martín Barbero, García Canclini y González, por ejemplo, no han realizado trabajo empírico - y casi nada de trabajo teórico- en audiencias televisivas en los últimos 15 ó 20 años.

Mientras que la supremacía de lo que podemos denominar, a falta de una mejor etiqueta, estudios culturales se explica por la importancia histórica de las aproximaciones críticas en la región, nuestra revisión muestra un rango muy bajo de diversidad e interés en otras tradiciones teóricas. La perspectiva de los efectos predominante en los Estados Unidos (agenda setting, análisis del cultivo, aprendizaje social, entre otros) está completamente ausente en el corpus analizado. En América 
Latina, por tanto, las audiencias televisivas no parecen estudiarse desde diferentes perspectivas teóricas como ocurre en Estados Unidos o Europa occidental.

Un hallazgo sorprendente es el número tan elevado de estudios que no mencionan explícitamente los fundamentos teóricos desde los cuales plantean sus preguntas de investigación o interpretan sus resultados: nueve estudios que representan 18\% de los reportes. Algunos de ellos son encuestas descriptivas que intentan ofrecer información básica sobre patrones de consumo (López, 2003, 2004). Otros han sido realizados por autores identificados con la perspectiva de estudios culturales (Covarrubias, 2001; Fuenzalida, 1992; Renero, 1996), pero por alguna razón no lo explicitan en la revisión de la literatura, en las preguntas de investigación o incluso en la interpretación de sus resultados. Dos estudios más (Bonilla, 1998; Huerta, 1999) abordan el importante tema de la violencia y la televisión, pero desde una base ecléctica o inexplícita.

\section{Falta de atención a lecturas ideológicas}

La tabla 6 muestra que aun siendo los estudios culturales la perspectiva teórica predominante en investigación empírica de audiencias televisivas, muy pocos trabajos abordan la pregunta central del impacto ideológico de los medios o la capacidad de las audiencias para negociar, resistir o rediseñar los contenidos ideológicos preferentes. Sólo 7 de más de 30 trabajos se basan en decodificación y lecturas de contenidos ideológicos (De Jesús y Patriota, 2007; Huerta, 2005; López Rivera, 1997; Lozano, 2003a, 2003b; Orozco, 1996; Saez, 2002), confirmando la preocupación de Morley (1997:121-137) sobre los investigadores culturalistas que con frecuencia pasan por alto cuestiones importantes como el poder y la ideología en el trabajo empírico.

Todos los demás estudios basados en estudios culturales se centran en el análisis y comprensión del papel de la televisión en la vida cotidiana de las audiencias (Cerda, 2001; Gendreau, 1997; Guadarrama, 1999; Marques y Rocha, 2006; Renero, 1995, 1996; Tufte, 2007; Yarto y Lozano, 2004) o en el rol de la familia y/o escuela como mediaciones de recepción televisiva (Aguiló, 2004; Bonin, 2005; Cornejo, 1992; Cornejo, Urteaga y Viveros, 1995; Fuenzalida, 1992; González Hernández, 2006; Huerta, 


\section{TABLA 6}

PORCENTAJE DE ARTÍCULOS POR TÓPICO ESTUDIADO

\begin{tabular}{lcc}
\multicolumn{1}{c}{ Tópico } & $\mathrm{f}$ & $\%$ \\
\hline Otros & 11 & 21.6 \\
Vida cotidiana & 8 & 15.7 \\
Mediación familiar & 7 & 13.7 \\
Otras mediaciones & 7 & 13.7 \\
Lecturas ideológicas & 7 & 13.7 \\
Consumo de medios & 4 & 7.8 \\
Género & 2 & 3.9 \\
Consumo de contenidos estadunidenses & 2 & 3.9 \\
Violencia y efectos & 2 & 3.9 \\
Credibilidad de las noticias & 1 & 2.0 \\
Total & 51 & $100 \%$ \\
\hline
\end{tabular}

2004; Lozano, 2000; Orozco y Viveros, 1996; Padilla, 2007; Renero, 1992, 1997; Uribe, 1993; Vink, 1992). Sin embargo, resulta importante señalar la relevancia de estos estudios (y muchos otros publicados en libros y revistas no contempladas en esta revisión), para el conocimiento de las formas en que la familia y la escuela filtran el consumo y decodificación de los programas televisivos en América Latina.

En contraste, otras áreas de gran interés cuando se estudia la recepción televisiva, como podrían ser la violencia, la credibilidad de las noticias, la apropiación de la agenda mediática o la adopción de versiones televisadas de la realidad, están escasamente estudiadas en la muestra. Esta cuestión se debe también a la falta de diversidad en las aproximaciones teóricas utilizadas por los investigadores latinoamericanos y podría ser incluso indicador de la falta de diversidad en la capacitación de los futuros académicos que estudian actualmente en los programas latinoamericanos de posgrado en comunicación.

\section{Avances en el frente metodológico}

McAnany y La Pastina concluyeron en su revisión de 26 trabajos de audiencias televisivas entre 1970 y 1993, que uno de los hallazgos más relevantes era que 
relativamente pocos [estudios] hacen referencia a sus metodologías, aparentemente porque las dan por un hecho. En general, los dos métodos empleados mas comúnmente son la encuesta y el estudio etnográfico de caso, aunque los grupos focalizados, la investigación-acción y otras metodologías también son utilizadas (1994:839).

Nuestra propia revisión de 51 estudios publicados en las revistas académicas más importantes de la región muestra que en los últimos 15 años un número significativo de investigadores latinoamericanos sigue haciendo poca o nula referencia a sus metodologías (tablas 7 y 8 ). Alrededor de $37 \%$ de los trabajos no explica o explica de manera incompleta su metodología, y alrededor de $30 \%$ de los estudios no incluye una sección formal de método en el cuerpo del trabajo (el porcentaje sube a $46 \%$ si también se incluyen los artículos que sí explicitan su metodología pero lo hacen en alguna otra sección, como en la revisión de la literatura). Este hallazgo muestra el largo camino por recorrer en América Latina en la capacitación de investigadores de la comunicación para lograr el rigor y la exigencia deseada en el trabajo empírico. Asimismo, este resultado alerta sobre la necesidad de que los editores y evaluadores de revistas académicas sean más estrictos al dictaminar y aprobar las investigaciones puestas a su consideración para ser publicadas.

Sin embargo, en un tono más optimista, los hallazgos demuestran un mejor balance entre las diferentes técnicas disponibles para la investigación de audiencias (tabla 9). La encuesta sigue ocupando un lugar importante, pero los métodos cualitativos se han diversificado con el uso de entrevistas a profundidad o focalizadas, grupos de discusión, etnografías y otras estrategias cualitativas. Un avance interesante es el creciente uso de la combinación de encuesta con métodos cualitativos dentro de un mismo estudio (Bonilla y Rincón, 1998; Chavero y Muñoz, 2005; De Jesús y Patriota, 2007; Gendreau, 1997; Lozano, 2000, 2003a; Renero, 1996, 1997; Tufte, 2007), demostrando una tendencia hacia la triangulación de los resultados. Este es un satisfactorio paso hacia adelante en una región donde los métodos cuantitativos habían sido, hasta hace poco, rechazados por su asociación "inherente" con el positivismo (cfr. Mattelart, 1976:165-180) y donde el divorcio académico entre los defensores de los métodos cualitativos y los seguidores de los cualitativos desgracidamente sigue estando presente en muchos casos. 


\section{TABLA 7}

PORCENTAJE DE ESTUDIOS QUE EXPLICAN SU METODOLOGÍA

\begin{tabular}{lcc}
\multicolumn{1}{r}{ Explican método } & f & $\%$ \\
\hline Completamente & 32 & 62.7 \\
Parcialmente & 14 & 27.5 \\
No explican & 5 & 9.8 \\
Total & $\mathbf{5 1}$ & $\mathbf{1 0 0 \%}$ \\
\hline
\end{tabular}

\section{TABLA 8}

PORCENTAJE DE ESTUDIOS CON SECCIÓN DE MÉTODO

\begin{tabular}{lcc}
\multicolumn{1}{c}{ Sección de método } & $\mathrm{f}$ & $\%$ \\
\hline Sí & 27 & 52.9 \\
No & 16 & 31.4 \\
Sí , pero con diferente nombre & 8 & 15.7 \\
Total & $\mathbf{5 1}$ & $\mathbf{1 0 0 \%}$ \\
\hline
\end{tabular}

\section{TABLA 9}

PORCENTAJE DE ESTUDIOS POR TÉCNICA

\begin{tabular}{lcc}
\multicolumn{1}{c}{ Técnica } & f & $\mathbf{\%}$ \\
\hline Encuesta & 13 & 25.5 \\
Entrevista a profundidad o focalizada & 13 & 25.5 \\
Encuesta y una técnica cualitativa & 10 & 19.6 \\
Grupos de discusión & 6 & 11.8 \\
Etnografía & 3 & 5.9 \\
Otras técnicas cualitativas & 3 & 5.9 \\
Datos secundarios & 2 & 3.9 \\
Historias de vida & 1 & 2.0 \\
Total & $\mathbf{5 1}$ & $\mathbf{1 0 0 \%}$ \\
\hline
\end{tabular}

De cualquier forma, el tamaño de las muestras continúa siendo una cuestión problemática, como lo apuntaban ya en su análisis McAnany y La Pastina (1994). La tabla 10 muestra que los tamaños de las muestras reportadas para las encuestas no son tan pequeños excepto en alrededor de $30 \%$ de los estudios basados en esta técnica (menos de 300 infor- 
mantes). Otro $70 \%$ de los artículos basados en esta misma estrategia cuantitativa oscilaban entre los 300 y los 3,000 informantes. Cabe señalar, sin embargo, que los únicos trabajos con 2000 informantes o más correspondían a los realizados por miembros de la Cátedra Televisa en el Tecnológico de Monterrey, Campus Monterrey (Cerda, 2002; Lozano, 2000, 2003a, Yarto y Lozano, 2004), reportando hallazgos de una muestra probabilística amplia en viviendas de Monterrey, Guadalajara, y ciudad de México sobre televisión y vida cotidiana. Otra muestra importante de 1,134 estudiantes de secundaria encuestados fue la realizada en Bogotá, Colombia, por la Universidad de la Sabana (López, 2003). Sin embargo, con excepción de los trabajos derivados de la Cátedra Televisa y algunos otros, muchas de las encuestas carecen de una discusión sobre la población y el tamaño de las muestras, los procedimientos para seleccionarlas, el diseño de investigación y otra información importante para alcanzar la calidad requerida en el trabajo de campo.

Las tablas 11 y 12 muestran el número de informantes en las entrevistas a profundidad o focalizadas así como de los grupos de discusión. En estos trabajos resulta más difícil evaluar la congruencia del tamaño de las muestras dada la flexibilidad y la variación de características y objetivos de cada uno de los estudios. Resulta problemático, sin embargo, que 32\% de los trabajos basados en entrevistas de tipo cualitativo no mencionen el número real de informantes que participaron en el estudio, y que en este mismo contexto, 13 de 26 artículos basados en técnicas cualitativas no expliquen o no aclaren completamente la metodología de su trabajo de campo. La falta de esta información imposibilita la evaluación de la relevancia, validez y calidad de los resultados reportados en los estudios. Es incomprensible la razón de las revistas para aceptar 50\% de estudios cualitativos que ignoran o no explican adecuadamente su metodología.

\section{DISCUSIÓN}

La investigación empírica de audiencias en América Latina sigue siendo escasa, a pesar de la fuerte presencia de la perspectiva de los estudios culturales, la tradición investigativa sobre telenovelas y las aportaciones globales de académicos como Martín Barbero, García Canclini y Orozco. La tendencia de los académicos latinoamericanos a optar por 


\section{TABLA 10}

TAMAÑO DE LAS MUESTRAS DE ARTÍCULO BASADOS EN ENCUESTAS

\begin{tabular}{lcc} 
Tamaño de muestra & $\mathrm{f}$ & $\%$ \\
\hline De100 a 299 & 5 & 29.4 \\
De 300 a 600 & 6 & 35.3 \\
De 1000 a 3000 & 6 & 35.3 \\
Total & $\mathbf{1 7}$ & $\mathbf{1 0 0 \%}$ \\
\hline
\end{tabular}

TABLA 11

NÚMERO DE INFORMANTES EN ENTREVISTAS CUALITATIVAS

\begin{tabular}{lcc} 
Número de informantes & $\mathrm{f}$ & $\%$ \\
\hline De 1 a 5 & 2 & 7.1 \\
De 10 a 20 & 6 & 21.4 \\
De 21 a 30 & 4 & 14.3 \\
De 31 a 60 & 2 & 7.1 \\
De 61 a 200 & 5 & 17.9 \\
No mencionan & 9 & 32.1 \\
Total & $\mathbf{2 8}$ & $\mathbf{1 0 0 \%}$ \\
\hline
\end{tabular}

TABLA 12

NÚMERO DE GRUPOS DE DISCUSIÓN

\begin{tabular}{lcc} 
Número de grupos & $\mathrm{f}$ & $\%$ \\
\hline $1-2$ & 2 & 33.4 \\
$4-5$ & 2 & 33.4 \\
7 & 1 & 16.7 \\
24 & 1 & 16.7 \\
Total & $\mathbf{6}$ & $\mathbf{1 0 0 \%}$ \\
\hline
\end{tabular}

ensayos teóricos en lugar de trabajo empírico, ya sea por falta de recursos económicos o por deficiente capacitación metodológica, ha permanecido presente en los últimos 15 años.

El análisis de 51 estudios de audiencias televisivas encontrados en 374 números de 17 de las revistas en comunicación más importantes de 
América Latina ofrece algunas bases sólidas para detectar los enfoques predominantes en géneros, tipos de audiencias, aproximaciones teóricas y técnicas de investigación que han caracterizado el trabajo empírico entre 1993 y el 2007. Uno de los hallazgos más relevantes es el desplazamiento de las telenovelas como foco central de los estudios de recepción en la región.

Mientras que $23 \%$ del número total de estudios sigue reflejando un interés hacia este género, en los últimos 15 años se ha mostrado una creciente preocupación por explorar el complejo ensamble de contenidos televisivos que consumen los diferentes segmentos de la audiencia y la recepción de programas de noticias. De cualquier forma, se requieren más estudios empíricos en géneros particulares que han adquirido relevancia por su cantidad y su consumo en la mayoría de los países de la región. Los reality shows, las series, las caricaturas y los programas deportivos, entre otros, han adquirido gran popularidad y han sido hasta ahora poco estudiados de manera particular. Es entonces sorprendente no encontrar trabajos cualitativos sobre la recepción y apropiación de géneros o programas individuales diferentes a la telenovela o los noticieros.

Para una región reconocida académicamente en el mundo por su interés en el imperialismo cultural y los efectos ideológicos de las industrias mediáticas locales y trasnacionales sobre las audiencias, es raro encontrar que sólo 7 de 51 estudios se concentraban en las lecturas y negociaciones ideológicas de los televidentes. Los culturalistas latinoamericanos parecen estar tomando el mismo camino impuesto por sus colegas en Estados Unidos y Europa, rechazando cuestiones como las de ideología y poder y centrando su atención en temas como vida cotidiana o mediaciones familiares y escolares en el proceso de ver televisión. Aunque la ausencia de teorías y aproximaciones positivistas puede ser considerada como favorable entre los académicos latinoamericanos, quienes posiblemente la interpretan como un intento de evadir otro tipo de dependencia de Estados Unidos, también resulta importante resaltar la uniformidad que genera, así como el empobrecimiento de debates, habilidades, aproximaciones y puntos de vista teóricos y metodológicos. La mayoría de las debilidades de la investigación empírica latinoamericana, en este campo, pueden provenir de esta homogeneidad y falta de diversidad en la enseñanza y revisión teórica de los programas de posgrado en comunicación en la región. 
El corpus estudiado en esta revisión muestra que la investigación empírica de audiencias televisivas en América Latina se ha vuelto más plural y sofisticada en el uso combinado de técnicas cuantitativas y cualitativas. Existe, sin embargo, un largo camino por recorrer con respecto al rigor metodológico, no sólo en el diseño de la investigación y el trabajo de campo, sino en la explicación requerida en los reportes de hallazgos con el fin de evaluar su calidad y relevancia.

Como se ha mencionado, este artículo forma parte de una investigación en proceso sobre estudios empíricos de recepción televisiva en América Latina. La inclusión de las revistas pendientes no contempladas en este corpus podría cambiar algunas de las conclusiones de esta revisión. Los trabajos analizados de Brasil, por ejemplo, son muy pocos debido a la escasez de revistas en texto completo en Revcom. Sin embargo, consideramos que los 51 estudios encontrados en 374 números de 17 revistas líderes de la región, representan una muestra válida para extraer inferencias iniciales sobre las tendencias, fortalezas y debilidades de esta línea de trabajo.

La investigación empírica de audiencias en América Latina tiene un gran potencial para contribuir significativamente en el conocimiento de los procesos de consumo, negociación y apropiación de contenidos televisivos en el marco mundial. El tamaño de la región, el valor incuestionable del pensamiento teórico local y la coexistencia de la tradición, la modernidad y la posmodernidad propia de los países latinoamericanos (García Canclini, 1990) podría conducir a conocer patrones de consumo y recepción considerablemente diferentes a los observados en la investigación actual de los países industrializados.

\section{Bibliografia}

CHONG, Blanca y Ornelas, José Luis (2006) "La mexicanidad y la preferencia por las películas estadunidenses del público de Torreón, Coahuila", ponencia presentada en el XVIII Encuentro Nacional AMIC, Morelia, México.

García Álvarez, Hugo (2007) "Consumo televisivo de origen estadounidense en el noreste de México", ponencia presentada en el XIV Encuentro Nacional CONEICC, Veracruz, México. 
GARCÍA CANCLINI, Néstor (1988) "Culture and power. The state of research", Media, Culture and Society, 10.

- (1990) Culturas híbridas. Estrategias para entrar y salir de la modernidad. México: Grijalbo.

GONZÁLEZ, Jorge (1987) "Los frentes culturales. Culturas, mapas, poderes y luchas por las definiciones legítimas de los sentidos sociales de la vida", Estudios sobre las Culturas Contemporáneas, (1)3.

GUTIÉRREZ, Alma E. (2006) "Consumo cultural de series televisivas norteamericanas en la zona noreste del país. El caso de Sex and the City entre estudiantes de una universidad privada de alto prestigio de la zona noreste del país", ponencia presentada en el XVIII Encuentro Nacional de Investigadores de la Comunicación AMIC, 2006, Morelia, México.

HinOJOSA, Lucila, Story, Lewis, Granat, M. y Garza, Rosalía (2005) "Consumo y apropiación de cine, video y televisión extranjero en el noreste de México", ponencia presentada en el XIII Encuentro Nacional CONEICC, Mérida, Yucatán, México.

FUENZALIDA, Valerio (2006) "Estudios de audiencia y recepción en Chile", Diálogos de la Comunicación (73).

JACKS, Nilda y Escosteguy, Ana C. (2006) "La investigación brasileña: Impases y desafíos", Diálogos de la Comunicación (73).

JENSEN, Klaus B. y RoSENGREN, Karl E. (1990) "Five traditions in search of an audience", European Journal of Communication, 5(2-3).

LOZANO, José Carlos (1990/1991) "Del imperialismo cultural a la audiencia activa: aportes teóricos recientes", Comunicación y Sociedad (10-11).

- (1996) "Media reception on the mexican border with the United States", en E. McAnany y K. Wilkinson (eds.), Mass media and free trade: NAFTA and the cultural industries, Austin, Texas, Estados Unidos: The University of Texas Press.

- (2001) "Consumo y lecturas negociadas de noticieros televisivos en Monterrey, Guadalajara y México, D.F", Oficios Terrestres, 7 (9/10), (Publicación de la Universidad Nacional de La Plata, Argentina).

- (2003) "Distanciamiento crítico frente a la televisión nacional mexicana", Revista de Estudios de Comunicación ZER, (14). 
- (2007) "Experiencias de investigación con alumnos de profesional y postgrado en el Tecnológico de Monterrey, México", Revista Universidad de Medellin, 42(83).

- (en prensa) "Consumo y apropiación de cine y televisión extranjeros por audiencias en América Latina: una revisión de su investigación", Comunicar, Revista iberoamericana de Comunicación y Educación, (30).

MATTELART, Armand (1976) "Críticas a la 'communication research", en J. Goded (ed.), Los medios de la comunicación colectiva, México: UNAM.

MARTÍN BARBERO, Jesús (1987) De los medios a las mediaciones: comunicación, cultura y hegemonia, Barcelona: Editorial Gustavo Gili.

— y Téllez, M. P. (2006) "Los estudios de recepción y consumo en Colombia", Diálogos de la Comunicación, (73).

MCANANY, Emile y La Pastina, Antonio (1994) "Telenovela audiences: A review and methodological critique of Latin American Research", Communication Research, 21(6).

MORLEY, David (1997) "Theoretical ortodoxies: Textualism, Constructivism and the 'New Ethnography' in Cultural Studies", en M. Ferguson \& P. Golding (eds.), Cultural Studies in Question. Thousand Oaks, California, Estados Unidos: Sage Publications.

OROZCO, Guillermo (1992) "Familia, televisión y educación en México", en G. Orozco (comp.), Hablan los televidentes: estudios de recepción en varios países, México, D.F.: Universidad Iberoamericana.

— y Padilla, M. Rebeca (2005) "Los estudios de recepción en México. Un itinerario", en Lozano, J.C. (ed), La comunicación en México: diagnósticos, balances y retos, Monterrey: Tecnológico de Monterrey, CONEICC.

SAINTOUT, Florencia y Ferrante, Natalia (2006) "Los estudios de recepción en Argentina hoy: rupturas, continuidades y nuevos objetos", Diálogos de la Comunicación, (73).

STRAUBHAAR, Joseph (1991) "Beyond media imperialism: assymetrical interdependence and cultural proximity", Critical Studies in Mass Communication (8). 


\section{Estudios revisados}

AGUILÓ Ignacio (2004) "El proceso de producción de sentido en televisión. Estudio de la codificación y decodificación de textos televisivos sobre piqueteros", Nombre Falso. http://www.nombrefalso. com.ar/index.php?

BONILLA, Jorge y Rincón, Omar (1998) "Violencia en pantalla. Televisión, jóvenes y violencia en Colombia", Diálogos de la Comunicación (53), http://www.dialogosfelafacs.net/dialogos_epoca/pdf/5304JorgeBonilla.pdf

BONIN, J.A. (2005) "Identidade étnica e recepção televisiva: revisitando dados de uma pesquisa empírica", FAMECOS, (28).

CERDA, Aída (2001) "Maneras de ver y usar la televisión: análisis por género de los hábitos de consumo televisivo de las audiencias mexicanas", Comunicación y Sociedad, (39).

Chavero, H. y García Muñoz, N. (2005) "Los hábitos de consumo televisivo de una comunidad extranjera: el caso de estudiantes latinoamericanos en Barcelona", Revista Zer (19).

CORNEJO, Inés (1992) "El psicodrama aplicado al estudio de la recepción familiar televisiva", Comunicación y Sociedad, (14-15).

- (1995) “Televisión sí, pero con orden”, Anuario Coneicc de Investigación de la Comunicación (2).

COVARRUBIAS, Karla (2001) "Hacia una nueva cultura televisiva: mirada de Mujer en la percepción de los públicos colimenses", Estudios sobre las Culturas Contemporáneas (7).

DALMONTE, E. (2006) "Dos efeitos fortes à hipótese de percepção do efeito de terceira pessoa: uma verificação empírica", Contemporanea, 4(1).

DE JESÚS, P. y, Albuquerque, N. S. (2007) “Telenovela malhação e cotidiano de prováveis extensionistas rurais em formação: um estudo de recepção junto a estudantes em São Lourenço da Mata - Pernambuco", Intercom 30(1).

DE LA GARZA, Yamile (1997) "Patrón de exposición televisiva de jóvenes de preparatoria de la ciudad de Monterrey", Anuario Coneicc de Investigación de la Comunicación (4).

DIEZ-MARTÍNEZ, E.; Miramontes, S. y Sánchez, M. (2000) "Las ocupaciones como elementos de la alfabetización económica y su reconocimiento a través de la televisión”, Comunicación y Sociedad (37). 
FUENZALIDA, Valerio (1992) “Telenovelas y desarrollo", Diálogos de la comunicación, (33), http://www.dialogosfelafacs.net/dialogos epoca/pdf/33-06ValerioFuenzalida.pdf

- (1996) "La apropiación educativa de la novela", Diálogos de la comunicación, (44)http://www.dialogosfelafacs.net/dialogos_epoca/ pdf/44-07ValerioFuenzalida.pdf

GENDREAU, Mónica (1997) "Globalización y medios de comunicación ¿hacia la aldea global?”, Anuario Coneicc de Investigación de la Comunicación, (4).

GONZÁLEZ, David (2006) "En busca del entretenimiento. Televisión y audiencia juvenil en la frontera norte", Comunicación y Sociedad (5).

GUADARRAMA, Luis Alfonso (1999) "Familias, telenovelas y futbol. Estudio de caso desde el enfoque sistémico", Anuario Coneicc de Investigación de la Comunicación (5).

HUERTA, Enrique, Garagarza, Ignacio y Villegas, Rebeca (1999) “Percepción de la violencia en televidentes del área metropolitana de Monterrey, México", Comunicación y Sociedad (36).

- y Cerda, Aída (2002) "Qué y cómo se ve televisión en México: oferta y consumo en tres áreas metropolitanas", Anuario Coneicc de Investigación de la Comunicación (9).

HUERTA, Enrique (2004) “'No le cambies a mi novela’. Dominación y negociación entre géneros en el acto de ver televisión", Comunicación y Sociedad (1).

- (2006) "Los programas de televisión estadounidenses y la percepción de la violencia en televidentes de Monterrey", Revista Zer, México (20).

—, Bañuelos, Berenice y Rodríguez, Alejandra (2006) "El rol de la televisión en la socialización política de los niños. Resultados preliminares", Anuario Coneicc de Investigación de la Comunicación, (13).

LETALIEN, B.L. (2005) "Pay televisión among low-income populations.Reflections on research performed in the Rio de Janeiro Favela of Rocinha", Contemporanea, 3(1).

LÓPEZ, Daniel F. (2003) "El consumo crítico de los medios de la juventud y el lenguaje de la discreción como propuesta", Palabra-Clave, (9), http://redalyc.uaemex.mx/src/inicio/ArtPdfRed.jsp?iCve=6490 0905\&iCveNum=3709 
- (2004) "Consumo de medios en estudiantes de secundaria de Bogotá. Una mirada desde cuatro escuelas de comunicación", Palabra-Clave, (10), http://redalyc.uaemex.mx/src/inicio/ArtPdfRed. jsp?iCve $=64901008 \& \mathrm{iCveNum}=3691$

LÓPEZ, L. (1996/1997) "Los Simpson y la reconstrucción social”, $R a-$ zón y Palabra, (5), http://www.razonypalabra.org.mx/anteriores/ n5/Simpsons.htm.

LOZANO, José Carlos (1994) "Recepción y uso de medios de comunicación en jóvenes fronterizos", Anuario Coneicc de Investigación de la Comunicación (1).

- (2000) "El género y el nivel socioeconómico como mediaciones en el consumo de noticieros televisivos en México", Revista Zer (9), http://www.ehu.es/zer/zer9/9lozano.html

- (2003a) "Consumo y lecturas negociadas de noticieros televisivos en Monterrey, Guadalajara y México DF”, Estudios sobre las Culturas Contemporáneas (9).

- (2003b) "Distanciamiento crítico frente a la tv nacional mexicana", Revista Zer (14), http://www.ehu.es/zer/zer14/distanciamiento14. htm.

MARTínEZ, Dora (1994) "Exposición a televisión, uso de medios impresos y razonamiento verbal entre jóvenes universitarios", Аnuario Coneicc de Investigación de la Comunicación (1).

MARTíNEZ, Francisco (1994) "La televisión y el aprovechamiento escolar de los niños de primaria en Monterrey, Nuevo León", Anuario Coneicc de Investigación de la Comunicación, (1).

MAYER, V. (2006) “A vida como ela é/pode ser/deve ser? O programa 'Aqui Agora' e cidadania no Brasil”, Intercom, 29(1).

ORTIZ, G., Ruiz, S. y Velásquez, C. (2002) "Inventar o reflejar la realidad. Un dilema que compromete la credibilidad informativa de los medios de comunicación en Colombia", Palabra-Clave, (7). http:// redalyc.uaemex.mx/src/inicio/ArtPdfRed.jsp?iCve $=64900702 \& \mathrm{iC}$ veNum $=3694$.

OROZCO, Guillermo (1996a) "La oferta televisiva y su percepción por jóvenes de la Ciudad de México", Anuario Coneicc de Investigación de la Comunicación, (3).

— (1996b) “'Hay que hacer algo, pero no somos los indicados'. Ám- 
bitos de mediación y supertemas en la televidencia de las noticias", Comunicación y Sociedad, (27).

PADILLA, Rebeca (2007) "Una nueva socialité llegó: historia de la recepción televisiva en la ciudad de Aguascalientes", Comunicación y Sociedad (7).

RENERO, Martha (1992) "De géneros televisivos y usos familiares", Comunicación y Sociedad (14/15).

- (1995) "Audiencias selectivas en el entorno. De la oferta multiplicada; el discurso materno acerca de los usos; de la televisión y otros medios", Comunicación y Sociedad, (24).

- (1996) "La diversión televisiva y el moderado placer de cada día: jóvenes, televisión y tiempo libre", Comunicación y Sociedad (28).

- (1997) "La influencia de la televisión en la juventud es algo más que una ideología. Aproximación cualitativa a las comunidades de significación familiar", Comunicación y Sociedad (29).

SÁEZ, N. (2002) "En torno al gran hermano", Nombre Falso. Electronic versión, http://www.nombrefalso.com.ar/index.php?pag=7

SALGUEIRO, A. y Rocha, S.M. (2006) “A produção de sentidos nos contextos de recepção: em foco o grupo focal”, Fronteiras, 8(1).

TILBURG, J. V. (1996) "La lectura de un texto televisivo (telenovela): Cuestiones metodológicas", Diálogos de la Comunicación, (44), http://www.dialogosfelafacs.net/dialogos_epoca/pdf/44-06JoaoVanTilburg.pdf

TUFTE, Thomas (2007) "Soap operas y construcción de sentido: mediaciones y etnografía de la audiencia", Comunicación y Sociedad, (8).

URIBE, Ana (1993) "La telenovela en la vida familiar cotidiana", Estudios sobre las Culturas Contemporáneas (15).

- (2005) "México imaginado. Recepción cultural, telenovelas e inmigrantes", Estudios sobre las Culturas Contemporáneas (11).

VEGA, Aimeé (2005) "Construyendo puentes: la identidad de género de los jefes de familia y la recepción televisiva”, Comunicación y Sociedad (4).

- (2004) “Amas de casa, televisión y participación política. México, elecciones 2003”, Revista Mexicana de Ciencias Politicas y Sociales (190). 
VINK, N. (1992) "La subcultura de la clase trabajadora y la decodificación de la novela brasileña", Diálogos de la Comunicación (34), http://www.dialogosfelafacs.net/dialogos_epoca/pdf/34-03NicoVink.pdf

YARTO, Consuelo y Lozano, José Carlos (2005) “Televisión, rutinas y vida cotidiana en mujeres de México, Guadalajara y Monterrey", Global Media Journal en Español (1), http:/gmje.mty.itesm.mx/ yarto_lozano.html. 